\title{
Cosmic ray observation at Antarctic Zhongshan station
}

\author{
J.L.Zhang', H.Lu, D.L.Zhang \\ Institute of High Energy Physic, Beijing 100049, China \\ E-mail: zhangjleihep.ac.cn
}

\begin{abstract}
By the end of 2014, a cosmic ray muon telescope consisting of two detectors which has 0.5 $\mathrm{m} \times 0.5 \mathrm{~m}$ area scintillator was installed at Antarctic Zhongshan Station. It is locate latitude and longitude coordinates of $69.4^{\circ} \mathrm{S}$ and $76.4^{\circ} \mathrm{E}$, respectively, at sea level. The vertical cutoff rigidity of cosmic ray protons at Zhongshan Station is $0.76 \mathrm{GV}$. The Zhongshan Station muon telescope data shows that there is a significant effect of pressure change with negative correlations. The observation data is sent to China by satellite link and is released through network.
\end{abstract}

\section{Introduction}

Cosmic-ray observation from satellites has a unique advantage: direct measurement of cosmic rays while avoiding the atmospheric effect; however, the small size of satellite-based detectors does not provide sufficient statistics in the high-energy region for real-time monitoring of the space environment. On the other hand, cosmic ray detectors on the ground can monitor the intensity of cosmic rays from $500 \mathrm{MeV}$ to $100 \mathrm{GeV}$ with sufficient statistics. They have been gathering important data on the space environment continuously for many years. Agreement has been reached in the cosmic ray research community that space and ground observations should be conducted simultaneously in such away so as to supply complementary data for a full understanding of the space environment [1].

Since 1957, ground observation has been carried out by means of NM-64 neutron monitors. The muon detectors complement neutron monitors by monitoring the cosmic ray modulation at slightly higher energy and provide measurements of numerous arrival directions of muons from a single location. A typical energy for a muon detector directional channel would be $\sim 50 \mathrm{GeV}$, as compared to $\sim 10 \mathrm{GeV}$ for neutron monitors. In recent years, some new ground-based muon monitors have been set up around the world to perform space environment studies using cosmic rays. Global Muon Detector Network (GMDN) was established in March 2006, when a hodoscope type cosmic ray detector $\left(9 \mathrm{~m}^{2}\right)$ in Kuwait was added to the previous network composed of the multidirectional detectors $\left(36 \mathrm{~m}^{2}\right)$ in Nagoya, Japan, a prototype muon detector $\left(4 \mathrm{~m}^{2}\right.$, expansion to $36 \mathrm{~m}^{2}$ in 2012) in Sao Martinho, Brazil, and a smaller one $\left(9 \mathrm{~m}^{2}\right)$ in Hobart, Australia [2].

The Yangbajing Cosmic Ray Observatory is located at Yangbajing, Tibet, China. It is locate at latitude and longitude coordinates of $30.11^{\circ} \mathrm{N}$ and $90.53^{\circ} \mathrm{E}$, respectively, at an altitude of $4300 \mathrm{~m}$ above sea level. The mean atmospheric pressure is $603 \mathrm{hPa}\left(600 \mathrm{~g} / \mathrm{cm}^{2}\right)$, and the vertical cutoff rigidity for cosmic rays is $14.1 \mathrm{GV}$. A new muon-neutron telescope was completed in Yangbajing in 2007 [3].

By the end of 2014, a cosmic ray muon telescope consisting of two detectors which has 0.5 $\mathrm{m} \times 0.5 \mathrm{~m}$ area scintillator was installed at Antarctic Zhongshan Station[4]. The observation data is sent to China by satellite link and is released through network. It is locate latitude and 
longitude coordinates of $69.4^{\circ} \mathrm{S}$ and $76.4^{\circ} \mathrm{E}$, respectively, at sea level. The vertical cutoff rigidity of cosmic ray protons at Zhongshan Station is $0.76 \mathrm{GV}$. Every 1 hour, the raw data of the mount telescope are transmitted from Zhongshan station in Antarctic to a server belonging to IHEP (Institute of High Energy Physics) in Beijing by satellite link. The raw data are analyzed and stored in a MySQL library. The server then publishes real-time data on the Internet. Users can either view the data graphically or download data (of resolution from every 60 seconds to daily averages) using a web browser from the address [5]. The data is regularly updated every hour. This data-publishing system is based on open standards and open-source software (HTML, Linux, MySQL, Apache and PHP).

This paper consists as follows. In Section 2, we briefly describes the set up of the Zhongshan Muon Telescope. The observed results for Forbush decrease (FD) are discussed in Section 3, and summary are drawn in Section 4.

\section{The Zhongshan Muon Telescope}

By the end of 2014, a cosmic ray muon telescope was installed at Antarctic Zhongshan Station. Trajectory calculations of cosmic ray particles for the site of Zhongshan Station were performed and determined effective cutoff rigidity and asymptotic directions for cosmic rays. Meteorological effects of Zhongshan Station muon telescope observational data are discussed.

\subsection{Setting}

Figure 1 shows a side view and logic block diagram of the Zhongshan Muon Telescope(ZSMT). As can be seen from this figure, ZSMT consists of two plastic scintillator detectors. Each scintillator is rectangular $50 \mathrm{~cm} \times 50 \mathrm{~cm}$ and $2 \mathrm{~cm}$ thick. Those two scintillators are vertically separated by $75 \mathrm{~cm}$ and can determine the arrival direction of muons. A photomultiplier tube (PMT) was placed $50 \mathrm{~cm}$ above the scintillator in to obtain a high uniformity of light collection. The 2" - diameter CR105-03 PMT used are produced in China. The inside of the stainless steel container holding each scintillator has been pasted with Tyvek to maximize light collection by the PMT. The output pulse from a PMT is sent to an emitter follower circuit, which is located in the phototube box. The pulse is transmitted to the amplifierdiscrimination-shaping module, which has been placed away from the PMT. The pulse from the detector is amplified and shaped into a digital signal by this module. When a particle passed through the upper and lower layers, an output signal from each detector is sent to the logic modules and a coincidence trigger pulse is generated by a FPGA (Field Programmable Gate Array). The trigger pulse are recorded by a scaler. Every $1 \mathrm{~s}$, the scaler data is send to the PC computer via an RS232 serial port. All these circuits are realized by one chip, the FPGA (Xilinx SPARTAN XC3S250E)[3]. For the convenience of conveying and installing, the lead plate between the two scintillators was not installed.

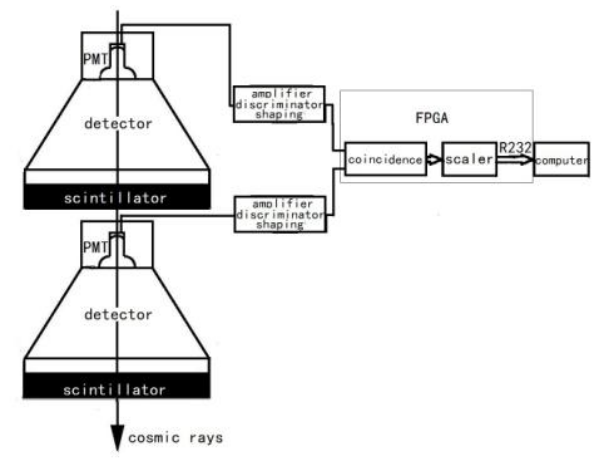

Fig. 1 Side view and logic block diagram of the Zhongshan Station muon telescope. 


\subsection{Zhongshan Station effective cutoff rigidity and asymptotic directions for cosmic rays}

Trajectory calculations of cosmic ray particles were performed using the FORTRAN program TJI95 provided by M.A. Shea [6]. As a result of these trajectory calculations we determined the calculated upper cutoff rigidity $\left(R_{U}\right)$, and the calculated lowest cutoff rigidity $\left(R_{L}\right)$, and an "effective cutoff rigidity" $\left(R_{C}\right)$. The effective cutoff rigidity was found by summing the allowed orbits through the penumbra: $R_{C}=R_{U}-\sum_{R_{L}}^{R_{U}} \delta R_{(\text {allowed })}$. Rigidity intervals of 0.01 GV were used for trajectories between $R_{U}$ and $R_{L}$ to provide a reasonable sample of the cosmic ray penumbra. Fig. 3 shows, for the zenith angle is $30^{\circ}$, the proton asymptotic direction for the five azimuthal angles.

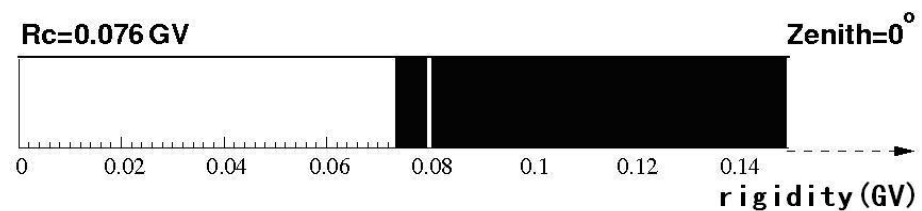

Fig. 2 Penumbra of the calculated vertical cutoff rigidity of cosmic ray protons at Zhongshan Station The effective cutoff rigidity is $0.076 \mathrm{GV}$

(Shadow area is the energy domain of particles that can enter Zhongshan Station).

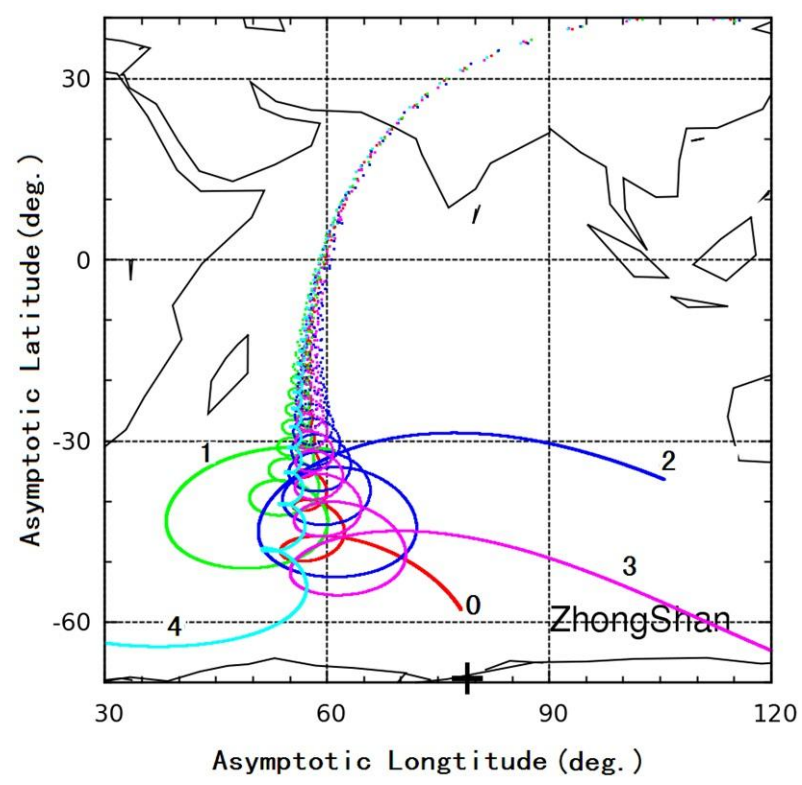

Fig.3 Asymptotic direction of the protons with rigidities of $0.1 \sim 100 \mathrm{GV}$ at Zhongshan Station. The zenith angle is $30^{\circ}$, and the azimuthal angle is northward (curve 1), eastward (curve 2), southward (curve 3), westward (curve 4) incident and normal incident respectively.

\subsection{Meteorological effects of Zhongshan Station muon telescope observational data}

Figure 2 shows hourly cosmic-ray counts and atmosphere pressure observed by the ZSMT, from the 1st to 30th of June 2015. It is observation that the pressure change is very strong at Zhongshan station. This change is from $963.85 \mathrm{hPa}$ to $1004.93 \mathrm{hPa}$ and change value is about 41 $\mathrm{hPa}$. During the 1 st to 30th of June 2015 , the change is from $596.78 \mathrm{hPa}$ to $607.48 \mathrm{hPa}$ and change value is about $10 \mathrm{hPa}$ at Yangbajing station, Tibet. It is necessary to a pressure correct of muon data for Zhongshan muon telescope. 


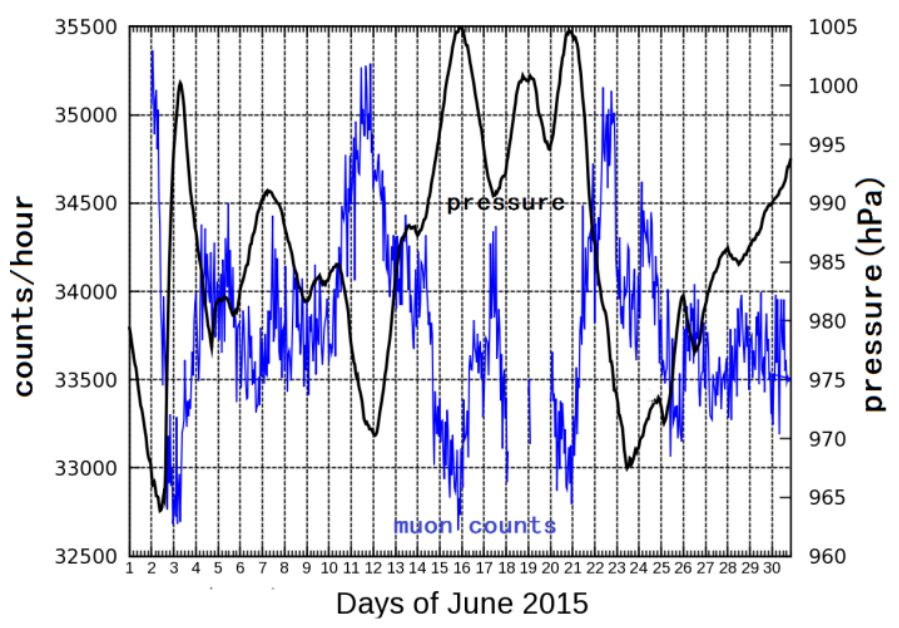

Fig. 4 Meteorological effect of Zhongshan muon telescope hourly counts.

From observed data from 1st to 30th June 2015, the mean pressure is 988 . The coefficient of pressure correct $(\beta)$ is -0.9295 . The pressure-corrected muon hourly counts are shown at Fig.3. Because the complexity of atmospheric environment at Zhongshan station, the pressurecorrected counts is not very effective.

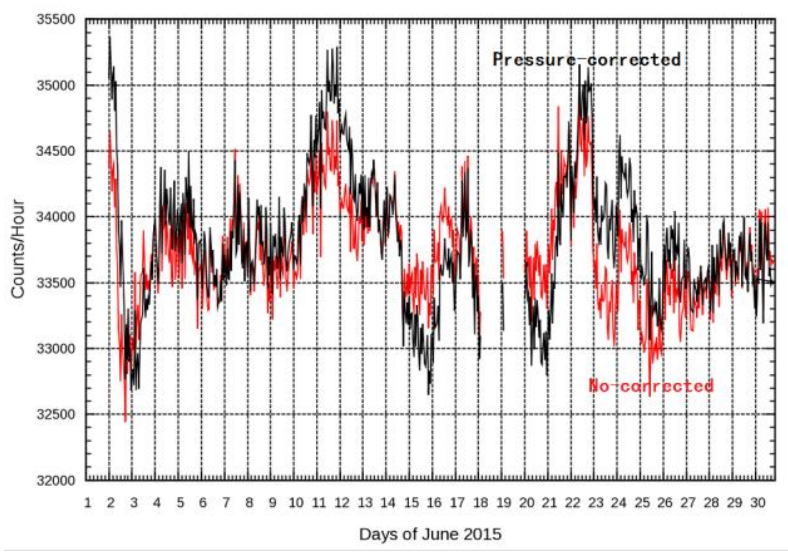

Fig. 5 Pressure-corrected muon hourly counts of the Zhongshan muon telescope from $1^{\text {st }}$ to $30^{\text {th }}$ June 2015.

\section{Observations for Forbush decrease in June 2015}

An impressive series of solar events occurred in June 2015. The Earth experienced a geomagnetic storm on June 22, 2015 due to the arrival of an Earth-directed CME from June 21. The CME originated at 2:36 UT on June 21[7]. Coronal material exploded from the sun with the velocity of $\sim 1366 \mathrm{~km} / \mathrm{s}$, arriving at the Earth at 5:45 UT on June 22. The same active region produced two other CMEs in the past few days, which were pushed (or compressed) along by the faster Earth-directed CME from June 21. The sudden storm commencement (SSC) identifies itself with the arrival of the ICME shock at 18:33 UT on June 22. This ICME are recorded from 02:00 UT at 23 on June to 14:00 UT on 24 [8]. A shock arrived at 13:29 UT on June 24 with an ICME from 25 June at 10:00 UT to 26 June at 06:00 UT. This ICME is related to the halo CME that was released at 18:36 UT on June 22.

During these fast ICMEs directed earthwards, a large FD event happens. We examine the sequence of solar events that occurred during 21-25 June and how they affect the counting rates of the Zhongshan muon telescope. 


\subsection{The galactic cosmic ray data}

We study the characteristics of the FD using neutron monitor (NM) data and muon telescope (MT) data for 22-26 June 2015.

The pressure corrected hourly rates (\%) from selected neutron monitors of the Bartol Research Institute are plotted in Fig.6. The South Pole neutron monitor is located at 90S. The McMurdo neutron monitor is located at 77.9S 166.6E. The mean value take from 1st to 20th, before IMF distribution. It is evident that the hourly rates for NMs in the Antarctic region, include the South Pole and McMurdo, shows evident FD onset (18:00 UT) before the shock arrival (SSC) at 18:33 UT on June 22 with 0.5 hours in advance. Each of the NMs shows very similar variations. Two neutron monitors began to decrease with the arrival of the shock and reached the minimum at 11:00 UT on June 23 while the Earth entered in the ICME. In association with a shock arrived at 13:29 UT on 24 and an ICME, this ICME is related to the CME that was released at 18:36 UT on June 22, the two neutron monitors decreased the counting rate again. On the other hand, a slight difference was recognized during decrease between the McMurdo and South Pole. The McMurdo had a pre-increase before the shock arrival while the South Pole had not. During the decrease time, the McMurdo shows an evident four-step decrease while the South Pole data did not show any obvious effect.

Fig.6 shows a plot of the hourly rates (\%) in the Vertical (V) for Nagoya muon telescope (MT). The hourly counting rate of the Nagoya muon telescope was taken from the Global Muon Detector Network (GMDN) data system of Shinshu University. The mean value was evaluated from 1 st to $20^{\text {th }}$ day before, of the IMF distribution. The Nagoya MT locates at $35.2 \mathrm{~N}$ 137E. The $\mathrm{R}_{\mathrm{m}}$ in the Vertical (V) values is $67 \mathrm{GV}$. The plot for Nagoya MT indicates that FD onset (19:00 UT) delayed $(\sim 0.5 \mathrm{~h})$ to the arrival of the SSC (18:33 UT) and reached the minimum at 7:00 UT on June 23 while the Earth entered in ICME. A pre-increase is visible obviously before FD onset. In association with a shock arrival at 13:29 UT on 24 and an ICME, the hourly counts of the Nagoya MT did not decrease again in comparison with the data of the NMs of South Pole and McMurdo.

As described in Section 2.3, the pressure change is very strong at Zhongshan station. It is necessary to a pressure correct of muon data for Zhongshan muon telescope. Because the count rate varies sharply as shown in Fig.5, a hourly counts 34300, a hourly counts before IMF distribution on June 22 , is taken as the hourly mean value. The cosmic ray hourly rates (\%) after pressure corrected of Zhongshan muon telescope is displayed in Fig.6. The plot for Zhongshan MT indicates that FD onset time (21:00 UT) is delayed ( $\sim 2.5 \mathrm{~h})$ with respect to the SSC (18:33 UT) and reach the minimum at 7:00 UT on June 23 while the Earth entered the ICME. The counts had a pre-increase in the leading to FD onset; in association with a shock arrivaled at 13:29 UT on 24 and an ICME, the hourly counts of the Zhongshan MT decreased simultaneous with the counting rate of the NMs of the South Pole and McMurdo.

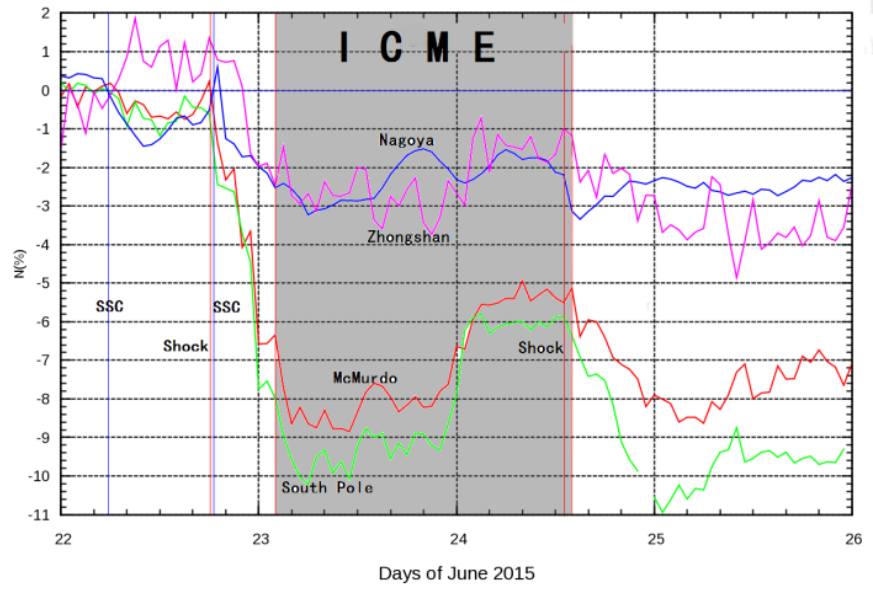

Fig. 6 The cosmic ray intensity variation (\%) hourly values of neutron monitor data and muon telescope. Data are plotted for June 22-26, 2015. 


\subsection{The solar wind measurements}

From June 21 to 25, 2015, a large FD event happens. The solar wind measurements from ACE and WIND are shown in Fig.7. The figure show the magnetic field intensity B, magnetic field $B_{x}, B_{y}, B_{z}$ (in the GSE coordinate system) component, solar wind proton density $n p$, solar wind proton temperature $T_{p}$, the solar wind proton speed $V_{p}$, and the geomagnetic activity index Dst.

The interplanetary shocks observed by the wind spacecraft: CfA Interplanetary Shock Database - Yearly Summary [9]. The times of shock is shown by solid vertical line. The time of the ICME associated geomagnetic storm sudden commencement (SSC), typically related to the arrival of a shock at Earth, take from the Service International des Indices Geomagnetique[10] . The time of SSC is shown by dashed vertical line.

The ICME contents from the list: Near-Earth Interplanetary Coronal Mass Ejections Since January 1996 compiled by Ian Richardson and Hilary Cane [8]. The ICME start time at 20200 UT of 23 and end at 1400 UT of 24. The gray shaded area indicates the interval of an ICME. The ICME shows an evidence of a rotation of the field direction, but lacks some other characteristics of a magnetic cloud, for example an enhanced magnetic field. Mean speed of the ICME is $610 \mathrm{~km} / \mathrm{s}$, based on solar wind speed observations during start and end times of the ICME. Maximum solar wind speed is $740 \mathrm{~km} / \mathrm{s}$ during the period from the disturbance of the shock to the trailing edge of the ICME. If the magnetic field within the ejecta is organized as a flux rope, then the magnetic field can be fitted by Higalgo's model [11]. By only having a quick look at the graph (Fig.7), we have found that this ejecta without any kinds of well-defined topology (no clear signatures: neither in the magnetic field components, which present a complex profile, nor in the plasma behavior). The profiles of the magnetic field component do not present the smooth and regular behavior expected for a flux-rope topology, but showed large fluctuating profiles during interval. The model cannot reproduce large fluctuations due to the irregular behavior of some magnetic field component; we can only fit smoothly the components for some of it. Another main consequence of those fluctuations in comparison with the theoretical mode appears in the magnitude of the magnetic field, where it is clearly seen that any flux-rope model has to fail for this kind of magnetic field structure. One can't see a magnetic flux rope topology embedded in ICME. This ICME is a Non-flux-rope ejecta.

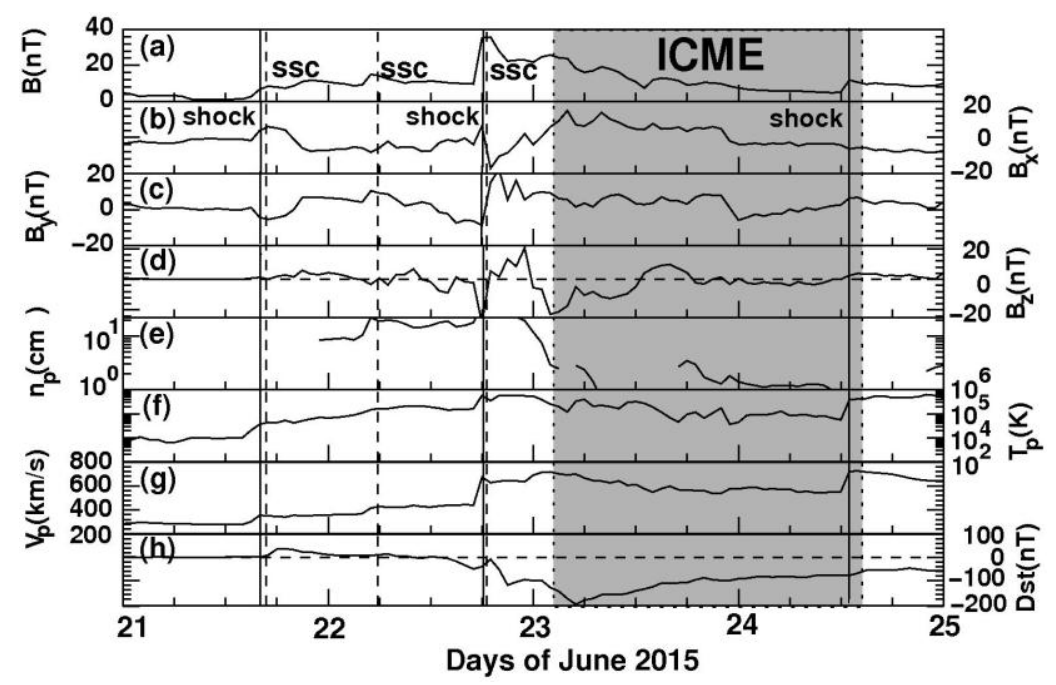

Fig. 7 Data are plotted for June 21-25, 2015.The figure from top to bottom shows hourly values of (a) the magnetic field strength $\mathrm{B}(\mathrm{nT})$ in the GSE coordinate system, (b) the magnetic field component $\mathrm{B}_{\mathrm{x}}$, (c) the magnetic field component $\mathrm{B}_{\mathrm{y}}$, (d) the magnetic field north-south component $\mathrm{B}_{\mathrm{z}}$, (e) the solar wind proton density $n_{p}$, (f) the solar wind proton temperature $T_{p}$, $(g)$ the solar wind proton speed $V_{p}$, (h) the geomagnetic activity index Dst. The start times of shock and SSC are shown by solid and dashed vertical lines, respectively. The shade gray area indicates the interval of an ICME. 
To study the fine structure of salient features, Fig.8 shows hourly plots of the magnetic field intensity $B_{Z}$, the solar wind proton speed $V_{P}$, the geomagnetic activity index Dst, the geomagnetic $\mathrm{K}_{\mathrm{P}}$ index and the intensity variation profiles of the Zhongshan muon telescope cosmic rays $\mathrm{N}(\%)$ with an expanded time scale for 24 hours (12UT June $22-24$ UT June 24, 2015). The positions of the shock and sharp storm sudden commencement (SSC) are marked; ICME lies to the right of the SSC (the shade gray area). The time of SSC (typically related to the arrival of a shock at Earth) is 22 18:33 UT.

The important interplanetary parameter for 'GCR-effectiveness' appears to be the enhanced and turbulent magnetic field. Scattering of cosmic-ray particles by an enhanced turbulent magnetic field in the sheath region between the shock front and the CME or magnetic cloud appears to be the most effective mechanism to produce FD in cosmic rays [12]. A sharp rise in the counts $(\mathrm{N})$ followd by a steep increase in $\mathrm{B}_{\mathrm{Z}}$ (Fig.8) and $\mathrm{V}_{\mathrm{P}}$ when the Earth enters the region between the SSC with leading edge of the ICME leading to the FD onset; this may be the region of turbulence. A sharp recovery occurs as the earth entrance in the ICME, a low GCR density region.

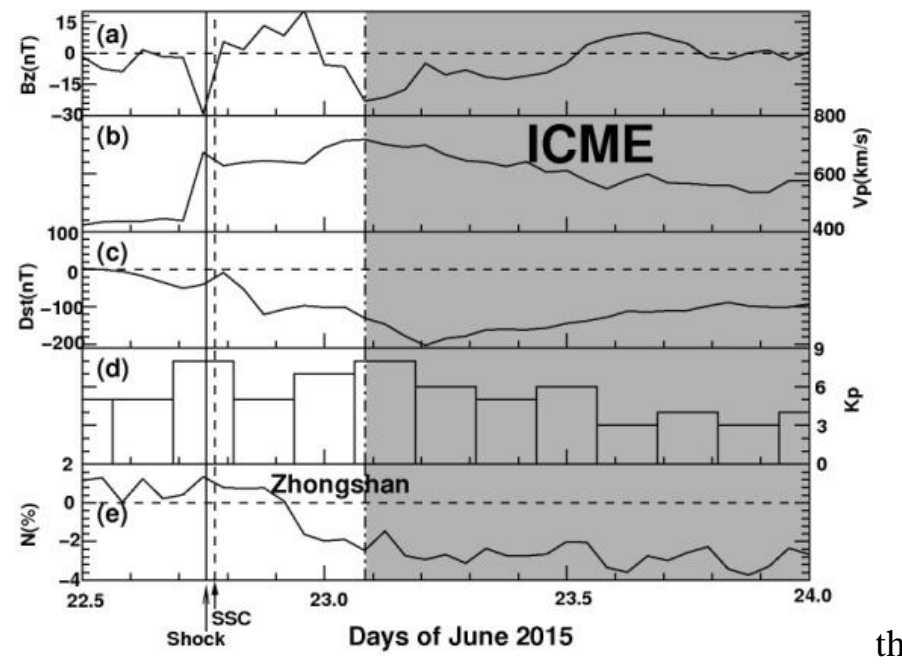

Fig. 8 Hourly values. Data are re-plotted for 12 UT June - 18 UT June 24, 2015. The figure from top to bottom shows hourly values of (a) the magnetic field strength $\mathrm{B}_{\mathrm{z}}(\mathrm{nT})$ in the GSE coordinate system, (b) the solar wind proton speed $V_{p}$, (c) the geomagnetic activity index Dst, (d) $K_{p}$ index , (e) the cosmic ray intensity variation(\%) profiles of Zhongshan muon telescope. The start times of shock and SSC are shown by solid and dashed vertical lines, respectively. The shade gray area indicates the interval of an ICME.

\section{Summary}

At Antarctic Zhongshan Station, a cosmic ray muon telescope was installed in 2014. An impressive series of solar events occurred in June 2015. From June 21 to 25, 2015, a large FD event happens. We study the characteristics of the FD using neutron monitor (NM) data and muon telescope (MT) data. The counting rates of the Zhongshan muon telescope indicate that FD onset (21:00 UT) delayed ( $\sim 2.5 \mathrm{~h})$ with respect to the SSC (18:33 UT) and reached the minimum at 7:00 UT on June 23 when the Earth entered in the ICME. In association with a shock arrival at 13:29 UT on 24 June and an ICME, the hourly counting rate of the Zhongshan MT decreased simultaneously with NMs of South Pole and McMurdo. The FD onset (19:00 UT) of Nagoya MT delayed $(\sim 0.5 \mathrm{~h})$ with respect to the SSC $(18: 33 \mathrm{UT})$. These results is different from the FD onset time of Nagoya MT that delayed $(\sim 5 \mathrm{~h})$ with respect to the SSC during 14 and 18 May, 2005[13]. A pre-increase before the shock arrival also was observed. The traditional model of FDs predicts that an ICME and its shock make reduction of the GCR intensity with a two-step profile [14]. However, this FD had a profile of four-step decrease. 
The traditional one- or two-step classification of FDs was inadequate to explain our study. Each FD must be studied as a unique event in the detailed context of its driving interplanetary conditions [15]. It was a four steps FD, probably due to the interaction of the faster CME on 21 June and the two slow CMEs in the past few days, which were pushed along by the faster the Earth-directed CME from June 21. The first step was caused by the shock. The second and the third one were caused by the entry into the extended sheath region. Finally, CRs entered in the enhanced region of the ejecta[16]

Due to a very small detection area, Zhongshan MT counting rate fluctuations are too large. It is further work to extend detection area. By the end of 2015, the Zhongshan muon telescope was enlarged to $1 \mathrm{~m} \times 1 \mathrm{~m}$ that consists of four $0.5 \mathrm{~m} \times 0.5 \mathrm{~m}$ detectors (Fig. 9). In addition, the observation data has achieved by satellite link real-time transmission [5], allowing real-time data exchange between Zhongshan station and cosmic ray observatories worldwide (for example, NMDB (real-time database for high resolution neutron monitor measurements) and

SPACESHIP EARTH[00] ), which promotes the joint study of the space environment
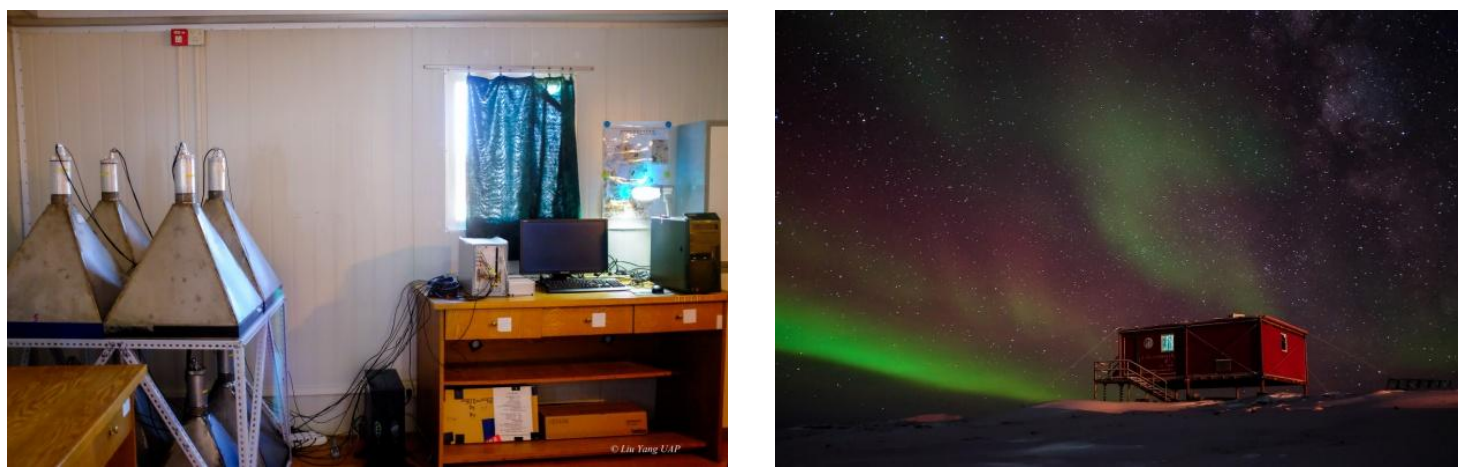

Fig. 9 The left figure shows the muon telescope in the room. The right figure shows the outdoor landscape of observation room.

\section{References}

[1] J. Bieber, et al, Proceedings of the 28th ICRC, Tsukuba, vol. 6, 2003, p. 3397

[2] M. Rockenbach, et al, Space Science Reviews 182: 1-18(2014)

[3] J. L. Zhang, et al, Nucl. Instr. and Meth. A, 623: 1030-1034 (2010)

[4] D.H. Huang, et al, Chin. J. space Sci., 36(6):897-903 2016

[5] http://ybjnm.ihep.ac.cn/muzhongshan8

[6] http://cdmc.gsfc.nasa.gov/modelweb/

[7] https://cdaw.gsfc.nasa.gov/CME_list/

[8] http://www.srl.caltech.edu/ACE/ASC/DATA/level3/icmetable2.htm

[9] https://www.cfa.harvard.edu/shocks/wi_data/

[10] http://isgi.unistra.fr/data_download.php

[11] M.A. Hidalgo, T. Nieves-Chinchilla and J.J. Blanco, Solar Physics, 284: 151-166(2013)

[12] Badruddin, Anand Kumar, solar Physics, 290: 1271-1283 (2015)

[13] H.S Ahluwalia, et al, Proceeding of $33^{\text {rd }}$ ICRC (Rio de Janeiro), 2013

[14] H. V. Cane, Space Science Reviews, 93:55-77 (2000)

[15] A.P.Jordan, et al, Journal of Geophysical Research, 116, A11103, doi: 10.1029/2011JA016791

[16] A.Papaioannou, et al, Journal of Physics: Conference Series 409(2013)012202, doi:10.1088/1742$6596 / 409 / 1 / 012202$ 